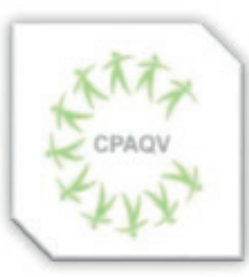

ISSN: 2178-7514
ARTIGO ORIGINAL

\section{UM EQUIPAMENTO ARTESANAL COMO ALTERNATIVA PARA O FORTALECIMENTO MUSCULAR DO PUNHO: UM ESTUDO ELETROMIOGRÁFICO}

\section{An artisanal equipment as an alternative to strengthening the wrist muscle: an electromyographic study}

Nayan Leonardo Sousa Lopes ${ }^{1}$ Walter de Aquino Vieira Filho', Márcio Clementino de Souza Santos ${ }^{3}$, Mônica Cardoso da Cruz ${ }^{4}$

Vol. 13| No. 2| Ano 2021

\title{
RESUMO
}

Dentre as diversas funções das mãos, destaca-se a de preensão, tornando a relação punho-mão e o equilíbrio muscular entre os flexores e extensores do punho determinantes para realizar tal tarefa. O objetivo do estudo foi avaliar a atividade EMG dos músculos flexores e extensores do punho em comparação às fases concêntricas e excêntricas do exercício, durante a utilização do Bastão de Enrolamento. A pesquisa teve início após aprovação do CEP da UNAMA e realizada entre 01/12/14 e 15/05/15 na FISIOCLÍNICA Bélem-PA. Participaram 19 voluntárias, entre 18 a 30 anos, sedentárias ou irregularmente ativas, destras, IMC $<25 \mathrm{~kg} / \mathrm{m}^{2}$ e hemodinamicamente estáveis. Para captação EMG foi utilizado o Eletromiógrafo de Superfície Miotool 400, com programa de captação Miograph 2.0. As voluntárias foram submetidas a uma avaliação e triagem, posteriormente ao teste de CMV e coleta de dados EMG utilizando-se do Bastão de enrolamento. $\mathrm{Na}$ amostra foi prefixado o poder do estudo $=85 \%$ e erro alfa $=5 \%$, as variáveis quantitativas mediante o teste t de Student, com nível de significância $=0.05$ para rejeição da hipótese nula. Para o processamento estatístico foi utilizado o BioEstat 5.4. Os resultados apontam uma efetiva ativação dos músculos flexores e extensores do punho durante os exercícios com o Bastão de Enrolamento, havendo significância ( $p<0.0001)$ nos testes: F.C x F.E; E.C x E.E; $\mathrm{F}^{*} \mathrm{D} \times \mathrm{F}^{*} \mathrm{E} ; \mathrm{E} * \mathrm{D} \times \mathrm{E} * \mathrm{E}$, os quais sugerem uma maior captação EMG na fase excêntrica do movimento e quanto maior for o braço de alavanca em relação ao punho analisado (direito).

Palavras-chave: Eletromiografia; Cinesiologia; Articulação do punho; Biomecânica

\begin{abstract}
Among the various functions of the hands, there is the hold, making the fist-hand relationship and muscular balance between the flexors and extensors of the determinants fist to accomplish such a task. The purpose of the study was to evaluate the EMG activity of the flexor and extensor muscles of the wrist compared to the concentric and eccentric phases of the exercise, while using the Bastão de Enrolamento. The research started after approval by the CEP UNAMA conducted between 12/01/14 to 05/15/15 at FISIOCLÍNICA Bélem-PA. Participated 19 volunteers, between 18 and 30 years, sedentary or irregularly active, right-handed, BMI $<25 \mathrm{~kg} / \mathrm{m}^{2}$ and hemodynamically stable. EMG pickup was used Surface Electromyograph Miotool 400, with 2.0 Miograph funding program. The volunteers were subjected to an evaluation and screening, after the CMV test and gathering EMG data using Bastão de Enrolamento. The sample was prefixed the study power $=85 \%$ and alpha error $=5 \%$, the quantitative variables by the Student $t$ test, with significance level of $=0.05$ for rejecting the null hypothesis. The statistical processing was used BioEstat 5.4. The results show an effective activation of the flexor and extensor muscles of the wrist during exercise with Bastão de Enrolamento, with significant ( $\mathrm{c}<0.0001)$ in the tests: F.C x F.E; E.C. x E.E; F*D x F*E; E*D x E*E, which suggest a higher uptake in EMG eccentric movement and the larger lever arm in relation to the analyzed handle (right).
\end{abstract}

Keywords: Electromyography; Kinesiology; Wrist Joint; Biomechanics.

1. Mestre em Ensino e Saúde: Educação Médica pelo Centro Universitário do Estado do Pará- CESUPA

2. Fisioterapeuta- Universidade da Amazônia- UNAMA

3. Doutor em Doenças Tropicais pela Universidade Federal do Pará- UFPA

4. Mestre em Fisioterapia pela Universidade Metodista de Piracicaba- UNIMEP

Autor de correspondência

Nayan Leonardo Sousa Lopes - E-mail: lopez.nayan@gmail.com

DOI: $10.36692 / \mathrm{v} 13 \mathrm{n} 3-2$ 


\section{INTRODUÇÃO}

A função padrão dos membros superiores (MMSS) não apenas inclui a capacidade de alcance e manuseio dos objetos, mas também a de direcionamento, preensão e manipulação dos mesmos $^{1}$. Tais componentes formam o alicerce para o desempenho das atividades de vida diárias (AVDs) com eficácia e precisão².

Os MMSS estão relacionados à movimentação das mãos, permitindo a elaboração de movimentos altamente complexos e finos ${ }^{3}$. Para a realização de tais funções, a articulação radiocarpal possui papel fundamental, sendo composta pelo rádio e os ossos escafoide e semilunar, configurando uma arquitetura do tipo condilóide, a qual permite os movimentos de flexão, extensão, os desvios ulnar, radial e circundação ${ }^{4}$. Além da radiocarpal, também contribuem de forma importante para os movimentos do punho, as articulações intercarpais, formadas entre os ossos do carpo, dentre os quais os já citados escafoide e semilunar, além do piramidal, psiforme, hamato, capitato, trapezóide, e trapézio 5 .

Existem aspectos relevantes que devem ser levados em consideração nas diversas formas de adaptação da mão, para desempenhar diferentes tipos de pegadas, que incluem a carga e o formato dos objetos, fatores que influenciam na preensão a ser utilizada ${ }^{6}$. Objetos cujos formatos se aproximam da anatomia das mãos proporcionam maior contato palmar, permitindo maior firmeza de preensão ${ }^{7}$.
Considerando-se a preensão palmar, a principal função cinesiológica da mão, é importante destacar a chamada relação punhomão como fator vastamente determinante no desempenho das atividades que envolvem este componente de gesto $^{8}$. Assim, o equilíbrio muscular entre os flexores e extensores do punho é necessário para o posicionamento ótimo da mão (posição neutra), de forma a contribuir diretamente para o ajuste da pegada?

Em relação às várias funções que esta articulação desempenha tais como, a de posicionar adequadamente as mãos para o ajuste da pegada e permitir alterações na localização e orientação das mesmas $^{10}$, a articulação do punho possui a função significativa de controlar a relação comprimentotensão dos músculos multiarticulares das mãos (intrínsecos das mãos) e do antebraço (flexor radial do carpo, flexor ulnar do carpo, flexor superficial e profundo dos dedos, extensor radial longo do carpo, extensor radial curto do carpo, extensor ulnar do carpo, extensor dos dedos indicador e mínimo), à medida que se ajusta em diversas atividades e formas diferenciadas de preensão ${ }^{11}$.

Devido à riqueza de estruturas e diferentes funções, vários métodos de fortalecimento muscular são desenvolvidos para os $\mathrm{MMSS}^{12}$, entretanto, a articulação do punho ainda carece de variações e alternativas para o seu desenvolvimento, uma vez que os MMSS possuem uma grande funcionalidade ${ }^{13}$. 
Os exercícios de fortalecimento muscular da articulação do punho frequentemente baseiamse em movimentos isotônicos (concêntricos e excêntricos), com alteres e barras, unilaterais ou simultâneos ${ }^{14}$. Visando o melhor desempenho relacionado às AVDs, buscou-se a adaptação de movimentos funcionais, semelhantes às práticas diárias $^{15}$, utilizando-se de um equipamento artesanal denominado Bastão de Enrolamento.

Como forma de justificar a utilização deste equipamento, a análise do movimento humano é fundamental para a compreensão dos estudos biomecânicos, clínicos e ocupacionais, pois permitem identificar as variações do movimento e fatores de riscos posturais presentes no cotidiano16. Desta forma, a Eletromiografia (EMG) tornou-se extremamente necessária para análise da ativação da função muscular ${ }^{17}$, durante a utilização deste equipamento.

Tendo em vista uma alternativa para o desenvolvimento muscular do punho, este estudo propõe a avaliar a atividade EMG dos músculos flexores e extensores do punho durante os exercícios de flexão e extensão utilizando-se do Bastão de Enrolamento.

\section{MÉTODO}

O equipamento foi construído utilizandose de $36 \mathrm{~cm}$ de material poliuretano inflexível de forma tubular de $50 \mathrm{~mm}$ de diâmetro, envolvido com dorso de papel crepado, tratado à base de poliuretano e adesivo de borracha para efeito de acabamento, tendo ao centro $(18 \mathrm{~cm})$ um orifício através do qual se transpõe uma corda trançada de polipropileno de $16 \mathrm{~mm}$ de diâmetro com $1 \mathrm{~m} 30 \mathrm{~cm}$ de comprimento (figuras 1, 2 e 3).

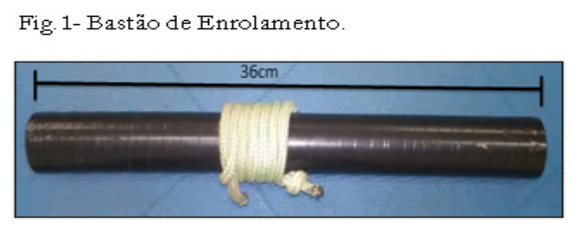

Fig.2- Diâmetro do equipamento.

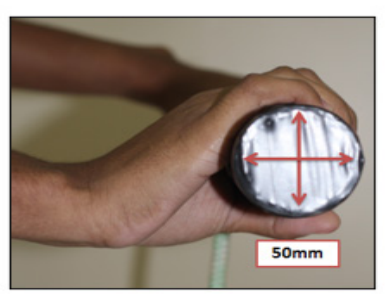

Fig.3-Centro do equipamento $(18 \mathrm{~cm})$.

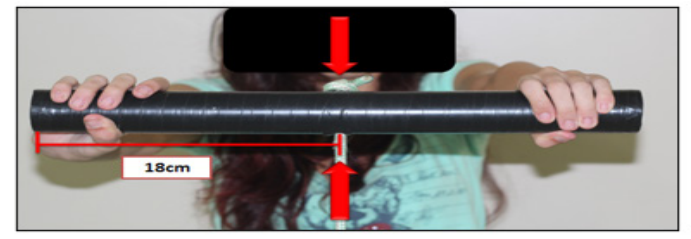


Este estudo teve início após a aprovação do Comitê de Ética em Pesquisa (CEP) da Universidade da Amazônia (UNAMA), $\mathrm{n}^{\circ}$ de protocolo 38106114, em atendimento a resolução 466/12 do Conselho Nacional de Saúde. Foi realizado no período de $01 / 12 / 14$ a 15/05/15, na Clínica Escola de Fisioterapia e Terapia Ocupacional (FISIOCLİNICA) Belém-PA. O cálculo amostral foi desenvolvido com base nos dados de um estudo piloto $(\mathrm{n}=3)$, recrutadas entre as estudantes da UNAMA, pela participação da fórmula abaixo definida pelo cálculo do tamanho da amostra ${ }^{18}$.

Equação 1- Tamanho da amostra.

$$
n=\frac{\left(\sigma 1^{2}+\sigma 2^{2}\right)(z 1-\beta)^{2}}{(\mu 2-\mu 1)^{2}}
$$

Para finalizar a amostra, no estudo piloto foi prefixado o poder do estudo $=0.85(85 \%)$ e o erro alfa $=0.05(5 \%)$. A partir da aplicação da fórmula acima foi estabelecido o tamanho amostral de $n=19$.

Foram incluídas na pesquisa voluntárias sem diagnóstico de doenças relacionadas aos MMSS, com idade entre 18 e 30 anos, destras, com padrão de vida sedentário ou irregularmente ativo, índice de massa corpórea (IMC) abaixo de $25 \mathrm{Kg} / \mathrm{m} 2$ e hemodinamicamente estáveis ${ }^{19}$. Ao todo 6 voluntárias foram excluídas, 1 apresentou doença relacionada aos MMSS, 4 estavam no seu período menstrual e 2 apresentaram histórico de cardiopatias.

\section{Avaliação e Triagem}

Com a finalidade de assegurar a homogeneidade da amostra, a partir da detecção das condições definidas como critérios de inclusão/exclusão, as voluntárias foram previamente submetidas a uma avaliação e triagem composta de três etapas:

Etapa 1- Entrevista: Aplicada a partir de um questionário elaborado pelos autores, contendo campos para preenchimento de dados pessoais, seguidos de 07 (sete) questões fechadas, com respostas padronizadas em sim/não, relacionadas às condições de: cardiopatias, pneumopatias, histórico de lesões relacionadas aos MMSS, sequelas motoras, período do ciclo menstrual, comprometimentos musculoesqueléticos e alterações hemodinâmicas (hipertensão ou hipotensão arterial).

Etapa 2- Avaliação do nível de atividade física: Para esta avaliação, foi utilizado através do IPAQ (Intenational Physical Activity Questionnaire). Essa versão é composta de questões abertas que permitem estimar o tempo dispendido por semana em diferentes dimensões de atividade física, como caminhada e esforços físicos de intensidades moderada e vigorosa ${ }^{20}$.

Etapa 3- Cálculo do IMC: Utilizandose de umas das ferramentas do Software para Avaliação Postural (SAPO), em sua versão 0.68, o qual foi desenvolvido pela Universidade de São Paulo (USP), tendo como referências a altura, peso, sexo e idade ${ }^{21}$, onde as voluntárias com valores acima de $25 \mathrm{~kg} / \mathrm{m}^{2}$ foram excluídas, já que 
o excesso de tecido adiposo interfere na eficácia da captação do sinal EMG ${ }^{22}$.

\section{Avaliação do sinal de Eletromiográfica} de Superfície (SEMG)

A captação do sinal de SEMG foi mediante a utilização de um Conversor Analógico Digital de quatro canais (Eletromiógrafo de Superfície e Biomecânica Miotool 400) com programa de captação de dados (Miograph 2.0 USB) com resolução de 16 bits $^{23}$ (figura 4). Os sinais foram coletados com frequência de amostragem de 2000 amostras/seg. por canal e captados por sensores diferenciais de superfície (SDS5000), e eletrodos circulares (Meditrace $\mathrm{Ag} / \mathrm{AgCl}$ ), com 10mm de diâmetro ${ }^{22}$.

Fig. 4 Eletromiógrafo de Superficie Miotool 400.

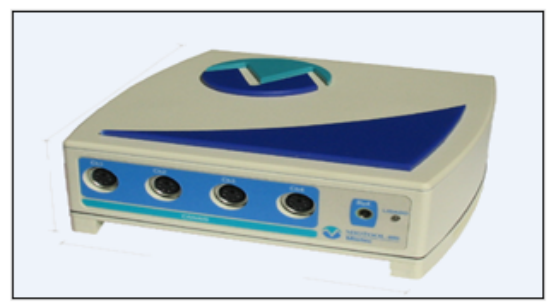

Teste de Contração Máxima Voluntária (CMV)

Para o teste de CMV a sala de avaliação foi adaptada para a utilização da Célula de Carga Miotec. As voluntárias foram orientadas a manterse sentadas, mantendo o alinhamento dos ombros e uma leve lordose lombar, com o intuito de manter a ativação da musculatura Paravertebral. $\mathrm{O}$ antebraço ficou apoiado sobre um suporte, com altura regulável de 85 a $125 \mathrm{~cm}$, mantendo um ângulo de $80^{\circ}$ a $90^{\circ}$ de flexão de cotovelo e ajustando a preensão palmar ao pegador unilateral de polia ${ }^{24}$ (figura 5).

Os eletrodos foram dispostos no ventre muscular ${ }^{25}$ do grupo flexor (canal 1) e extensor do punho (canal 2). Para a marcação anatômica do eletrodo de referência, o maléolo medial (tibial) foi escolhido como o referencial e o canal 3 ajustado à célula de carga.

Fig. 5- Teste CMV.



A distância dos eletrodos foi de $5 \mathrm{~cm}$ em relação aos epicôndilos (medial e lateral do úmero) e de $2 \mathrm{~cm}$ entre eles, onde o polo (+) foi proximal e o polo (-) distal em relação a disposição longitudinal dos músculos, tendo como referência proximal o epicôndilo lateral do úmero (para os músculos extensores) e o epicôndilo medial do úmero (para os músculos flexores) ${ }^{22}$.

Durante o teste, as voluntárias mantiveram uma contração máxima contínua durante 5seg, repetindo esta avaliação 3 vezes, onde foram captados os sinais de SEMG e definida a média mediante as 3 contrações. A flexão do punho foi avaliada inicialmente e posteriormente a extensão (estabelecido pelos autores). 


\section{Coleta de dados com o Bastão de Enrolamento}

Durante a avaliação, as voluntárias foram orientadas a manter-se de pé, com os MMSS elevados à altura do ombro, mantendo uma flexão de ombro de $90^{\circ}$, cotovelos em extensão, antebraço pronado, punho em posição neutra e adequando a preensão palmar nas extremidades do equipamento (figura 7). Durante a utilização do equipamento, as voluntárias seguiram rigorosamente as orientações dos pesquisadores, com o intuito de manter a mecânica correta do exercício e minimizar movimentos compensatórios, para o registro de melhores resultados EMG.

Fig. 8- Posicionamento

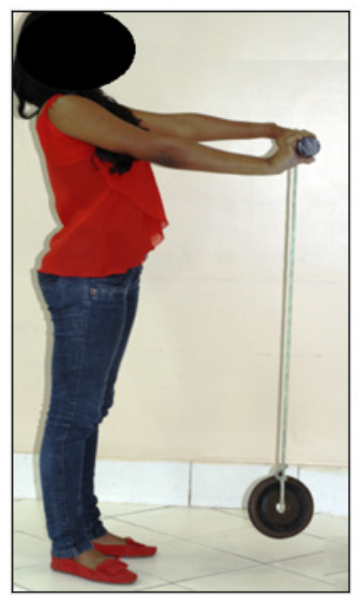

Para as próximas etapas, os eletrodos do canal 1, 2 e o canal específico para o eletrodo de referência utilizaram os mesmos parâmetros de posicionamento e distribuição citados no teste de CMV e a carga utilizada para todas as coletas de dados com o equipamento foi de $1 \mathrm{~kg}$ (aplicada a partir dos resultados do estudo piloto, em consequência da ativação EMG considerável constatada com tal resistência). Todos os testes foram realizados apenas para o punho direito (critério de inclusão), onde a ordem foi:

- $\quad$ Análise dos dados EMG dos flexores do punho em relação à sua fase concêntrica e excêntrica;

- $\quad$ Análise dos dados EMG dos extensores do punho em relação à sua fase concêntrica e excêntrica;

- $\quad$ Análise dos dados EMG quanto à aproximação ou afastamento da carga em relação ao punho direito.

\section{Normalização do SEMG}

A normalização do sinal de SEMG tem sido descrita como crucial para comparações entre diferentes sujeitos, medidas, grupos musculares ou estudos científicos ${ }^{26}$ e existem vários métodos de normalização do sinal de SEMG27. Em resumo, normalizar significa uma tentativa de diminuir as assimetrias ou diferenças EMG entre diferentes sujeitos, favorecendo a uma análise comparativa entre $\operatorname{eles}^{28}$.

Umas das medidas utilizadas para a análise da captação EMG é o Root Mean Square (RMS). Esta forma de registrado não requer retificação, pois a onda de amplitude do sinal é elevada ao quadrado, gerando valores positivos da captação do sinal ${ }^{29}$, destacado na fórmula abaixo:

Equação 2- Conversão do sinal em RMS.

$$
R M S=\sqrt[2]{\frac{1}{N}} \sum_{i=1}^{N} x^{2}{ }_{i}
$$


Cada procedimento foi repetido três vezes e a média dos valores RMS obtidos para cada grupo muscular (flexores e extensores do punho) em seu respectivo período (excêntrico e concêntrico) foi registrada, assim como os dados relacionados à influência da aproximação ou afastamento da carda em relação ao punho direito.

Após coletado dos valores RMS, foi realizada a seleção de intervalo (seleção da área de início e fim da captação EMG) durante o período de ativação muscular, com uma janela de seleção de 5 segundos, gerando valores da mínima, média, máxima e desvio padrão (ferramenta estatística do Miograph 2.0).

Utilizando-se dos dados obtidos, foi proposta a seguinte equação:

Equação 3- Normalização do sinal EMG.

\section{$R M S n=\frac{R M S b x 100}{C M V}$}

Onde:

- RMSn: Valor de RMS normalizado para cada grupo muscular, associado ao seu respectivo período de captação do sinal EMG (concêntrica, excêntrica ou deslocamento da carga);

CMV: Valor da média de RMS durante o período de contração máxima voluntária para cada grupo muscular (flexores e extensores do punho);

\section{Procedimentos estatísticos}

Para avaliar a atividade EMG dos músculos flexores e extensores do punho em uma amostra de $n=19$ voluntárias foram aplicados métodos estatísticos descritivos e inferenciais. As variáveis quantitativas foram apresentadas por medidas de tendência central e de variação. A comparação entre a atividade muscular (concêntrica e excêntrica; deslocamento da carga para a direita e para esquerda) foi realizada pelo teste t de Student para amostras pareadas30. Foi previamente fixado o nível de significância alfa $=0.05$ para rejeição da hipótese de nulidade. $\mathrm{O}$ processamento estatístico foi realizado utilizandose do software BioEstat versão 5.4.

\section{RESULTADOS}


Tabela 1 - Caraterização da amostra $(\mathbf{n}=19)$.

\begin{tabular}{llll}
\hline Variáveis & Média $\pm \mathbf{d} \mathbf{p}$ & Min. - Max. & Dado em: \\
\hline Idade & $20.5 \pm 2.4$ & $18-19$ & anos \\
MC & $53.8 \pm 1.6$ & $51.1-56.4$ & $\mathrm{Kg}$ \\
Estatura & $159.1 \pm 5.7$ & $151-172$ & $\mathrm{~cm}$ \\
IMC & $19.5 \pm 3.6$ & $14.3-24.5$ & $\mathrm{Kg} / \mathrm{m}^{2}$ \\
PAIS & $126.8 \pm 10.1$ & $110-140$ & $\mathrm{mmhg}$ \\
PAID & $73.6 \pm 6.8$ & $60-80$ & $\mathrm{mmhg}$ \\
PAFS & $130.5 \pm 8.5$ & $120-140$ & $\mathrm{mmhg}$ \\
PAFD & $76.3 \pm 4.9$ & $70-80$ & $\mathrm{mmhg}$
\end{tabular}

$\overline{\mathbf{M C}}=$ Massa corporal; $\mathbf{I M C}=$ Índice de massa corporal; PAIS $=$ Pressão arterial inicial sistólica; PAID $=$

Pressão arterial inicial diastólica; PAFS $=$ Pressão arterial final sistólica; $\mathbf{P A F D = ~ P r e s s a ̃ o ~ a r t e r i a l ~ f i n a l ~ d i a s t o ́ l i c a ; ~}$

Méd. $=$ Média aritmética; $\mathbf{d} \mathbf{p}=$ Desvio padrão; Min. $=$ Mínimo; Max. $=$ Máximo.

A amostra constituiu 19 voluntárias, entre $\quad 73,7 \%$ ) ou irregularmente ativas (5 voluntárias ou 18 e 29 anos de idade (20.5 \pm 2.4 ), destacando: MC 26,3\%) de acordo com o IPAQ.

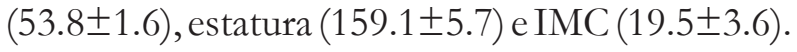

Tabela 2- Dados após a normalização Consideradas estáveis hemodinamicamente, do sinal EMG, analisando os movimentos de

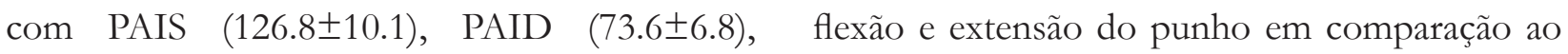
PAFS (130.5 \pm 8.5$)$ e PAFD (76.3 \pm 4.9$)$, sendo período concêntrico e excêntrico do movimento, classificadas como sedentárias (14 voluntárias ou mediante a utilização do Bastão de Enrolamento.

\begin{tabular}{|c|c|c|c|c|}
\hline \multicolumn{5}{|c|}{ Dados da captação EMG convertidos em RMSn (\%) } \\
\hline Testes & FC & FE & EC & EE \\
\hline Mínimo & 19.9 & 26.3 & 24.4 & 33.8 \\
\hline Máximo & 49.4 & 55.8 & 56.2 & 80.1 \\
\hline Amplitude Total & 29.5 & 29.5 & 31.8 & 46.3 \\
\hline Mediana & 31.8 & 38.1 & 37.7 & 48.7 \\
\hline Primeiro Quartil & 28.4 & 34.9 & 32.1 & 38.1 \\
\hline Terceiro Quartil & 38.9 & 45.3 & 48.6 & 54.1 \\
\hline Desvio Interquartilico & 10.5 & 10.4 & 16.5 & 16.0 \\
\hline Média Aritmética & 34.4 & 40.5 & 39.8 & 49.0 \\
\hline Desvio Padrão & 8.3 & 8.5 & 10.0 & 12.7 \\
\hline p-valor & \multicolumn{2}{|c|}{$<0.0001^{*}$} & \multicolumn{2}{|c|}{$<0.0001^{*}$} \\
\hline \multicolumn{5}{|l|}{ FTeste $t$ de Student } \\
\hline \multicolumn{5}{|c|}{$\begin{array}{l}\text { RMSn= RMS normalizados em percentual; FC = Teste de captação EMG durante o movimento de flexão do } \\
\text { punho em relação ao seu período concêntrico; FE = Teste de captação EMG durante o movimento de flexão } \\
\text { do punho em relação ao seu período ex cêntrico); EC = Teste de captação EMG durante o movimento de } \\
\text { extensão do punho em relação ao seu período concêntrico; EE = Teste de captação EMG durante o movimento }\end{array}$} \\
\hline
\end{tabular}


$\mathrm{Na}$ avaliação da atividade EMG dos músculos flexores do punho, no tocante à fase concêntrica e excêntrica, verificou-se que durante período excêntrico (40.5 $\pm 8.5 \mathrm{RMSn} \%)$ uma maior captação EMG foi registrada em comparação à fase concêntrica do movimento

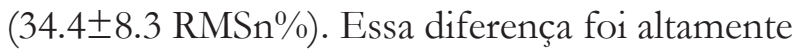
significante (p-valor $<0.0001 *)$.

Em relação ao grupo muscular extensor, a respeito da fase concêntrica e excêntrica,

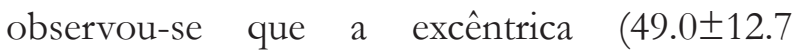
RMSn\%) apresentou maior atividade EMG que a concêntrica $(39.8 \pm 10.0 \mathrm{RMSn} \%)$, dados semelhantes aos do grupo muscular flexor do punho. Essa diferença foi altamente significante (p-valor $\left.<0.0001^{*}\right)$.
Estes resultados sugerem que tanto no movimento de flexão do punho, quanto no de extensão, o período excêntrico evidencia uma maior atividade muscular (influenciando numa maior captação EMG) se comparado à fase concêntrica do exercício durante a utilização do Bastão de Enrolamento.

Tabela 3- Dados após a normalização do sinal EMG, analisando a influência do enrolamento da corda para o lado direito ( $F * D$ e $\left.\mathrm{E}^{*} \mathrm{D}\right)$ e para o lado esquerdo $\left(\mathrm{F}^{*} \mathrm{E}\right.$ e $\left.\mathrm{E}^{*} \mathrm{E}\right)$ em relação ao punho direito, mediante à utilização do Bastão de Enrolamento na fase concêntrica, no tocante dos movimentos de flexão $\left(\mathrm{F}^{*} \mathrm{D}\right.$ e $\left.\mathrm{F}^{*} \mathrm{E}\right)$ e extensão $\left(\mathrm{E}^{*} \mathrm{D}\right.$ e $\left.\mathrm{E}^{*} \mathrm{E}\right)$ do punho.

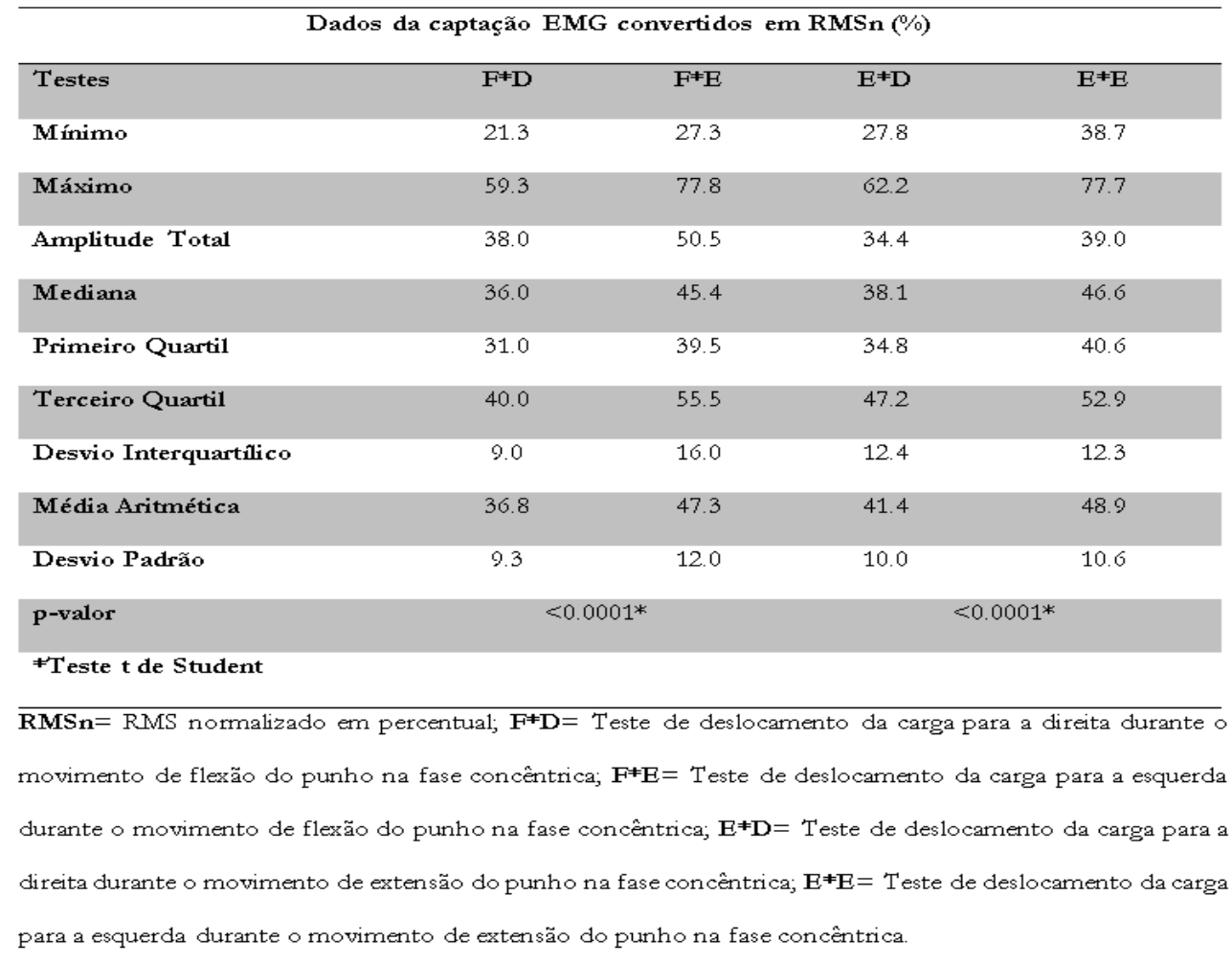


$\mathrm{Na}$ avaliação da atividade EMG dos músculos flexores, no tocante à lateralidade (deslocamento da carga) para a direita ou para a esquerda, verificou-se que o movimento para a esquerda, período de maior braço de alavanca,

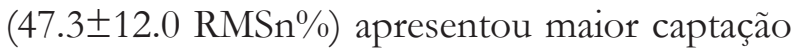
EMG comparado ao movimento para a direita (36.8 $\pm 9.3 \mathrm{RMSn} \%)$. Essa diferença foi altamente significante ( $\mathrm{p}$-valor $<0.0001 *)$.

$\mathrm{Na}$ análise da captação EMG dos músculos extensores do punho, em relação à lateralidade para a direita ou para a esquerda, definiu-se que o movimento para a esquerda, período de maior braço de alavanca, (48.9 \pm 10.6 RMSn\%) apresentou maior atividade EMG que o movimento para a direita (41.4 $\pm 10.0 \mathrm{RMSn} \%)$. Essa diferença foi altamente significante (p-valor $<0.0001 *)$.

Em todos os resultados observou-se uma relação direta em relação ao braço de alavanca (maior o braço de alavanca $=$ maior ativação muscular). Todos os testes de lateralidade $\left(F^{*} E ; F^{*} E ; E^{*} D ; E^{*} E\right)$ foram avaliados em seu período concêntrico, fase em que se destacam os movimentos de afastamento (enrolamento da corda para esquerda) ou aproximação da carga (enrolamento da corda para direita), já que no seu período excêntrico a carga move-se em direção ao centro do equipamento, tornando assim irrelevante a avaliação nesta fase.

\section{DISCUSSÃO}

O propósito deste estudo foi de avaliar atividade EMG dos músculos flexores e extensores do punho mediante a utilização do Bastão de Enrolamento. Os resultados da captação EMG mostraram que há uma ativação considerável dos grupos musculares analisados durante a execução dos exercícios, nas respectivas fases de flexão e de extensão com a mediana dos sinais normalizados (RMSn) variando entre $31,8 \%$ e $48,7 \%$ da média dos sinais obtidos nas coletas de CMV (correspondente aos 100\% de ativação muscular).

Considerando que os percentuais de atividade EMG descritos foram obtidos perante a execução dos exercícios com resistência de $1 \mathrm{~kg}$, tais achados permitem inferir que o equipamento Bastão de Enrolamento é efetivo para o trabalho de fortalecimento dos músculos flexores e extensores do punho, podendo ser utilizado como recurso alternativo para o citado objetivo. Os dados EMG demonstram ainda que no período excêntrico houve maior atividade muscular em comparação ao concêntrico, o que foi evidente tanto no movimento de flexão quanto no de extensão do punho.

A respeito do movimento de lateralidade (enrolamento da corda para direita ou para esquerda e/ou afastamento ou aproximação da carga), é importante ratificar que este teste, assim como os demais, teve como referência o punho direito (destacado nos critérios de inclusão). Tal 
análise registrou que durante a lateralidade à esquerda, a carga move-se numa direção oposta ao punho direito, aumentando gradualmente o braço de alavanca, tornando a ativação EMG evidente neste período.

Além da análise efetiva do período excêntrico, foi definido que quanto maior for o braço de alavanca durante o movimento (flexão ou extensão do punho) maior é a atividade muscular, o que ocorreu quando o enrolamento da corda foi realizado para o lado esquerdo, correspondendo ao afastamento da carga em relação ao punho direito.

Tratando-se deste aspecto, o período concêntrico, fase que ocorre o "enrolamento da corda", é o momento que evidencia o deslocamento da carga, o que tornou fundamental o estudo biomecânico durante a utilização do Bastão de Enrolamento. $\mathrm{Na}$ avaliação foi comprovada uma alta significância, o que definiu necessária a estimação da execução dos exercícios para ambos os lados, o que aponta à necessidade do desenvolvimento de novas pesquisas sobre esta temática, a fim de minimizar assimetrias musculares, com o intuito de aperfeiçoar o objetivo de fortalecimento muscular deste equipamento, confirmado conforme os resultados.

Nos estudos de Ide, Ramari, Muramatsu, et al. $^{31}$, é destacado que vários fatores podem influenciar ou alterar a captação do SEMG, tais como: formato, posicionamento, tipo de eletrodo e análise de diferentes grupos musculares, havendo uma parcela de ensaios EMG que abordam a fase concêntrica sendo mais efetiva. Os autores descrevem que durante o período concêntrico do movimento, há maior ativação muscular, concluindo numa maior ativação EMG, o que diverge dos resultados desta pesquisa.

Carpentier, Duchateau e Hainaut ${ }^{32}$ descrevem que um movimento realizado de forma lenta proporciona um maior recrutamento ativo da musculatura alvo (fato destacado na fase excêntrica da coleta), enquanto um movimento mais rápido gera menor captação EMG. Estas argumentações esclarecem os resultados desta pesquisa, uma vez que foi evidente um movimento mais acelerado durante a fase concêntrica do exercício e lento na excêntrica.

De acordo com os autores acima, movimentos realizados em diferentes velocidades (lenta, média e rápida) são aspectos altamente influentes na eficácia da captação EMG e alterações significantes ocorrem quanto à ativação muscular e característica da manutenção da onda de pico.

Levando-se em consideração tais resultados, o que pode ter influenciado numa divergência direta em relação a este tema? Blackburn, Mynark, Padua e Guskiewicz ${ }^{33}$ afirmam que há uma grande controvérsia quanto aos resultados em estudos EMG e que vários fatores podem influenciar na captação do sinal. Os autores afirmam que os tipos de fibras musculares podem influenciar no tipo de onda EMG e que diferentes grupos musculares podem apresentar-se de maneira distinta. 
A respeito desta afirmação, o SENIAM ${ }^{25}$ (órgão regulamentador de normas e diretrizes para estudos EMG) descreve de forma sucinta todos os parâmetros a serem utilizados para cada grupo muscular relacionado à captação EMG (como tamanho adequado e posicionamento correto dos eletrodos), entretanto, em relação aos flexores e extensores do punho não há especificações exclusivas para tais músculos. Para os MMSS podem-se observar apenas direcionamentos aos músculos tríceps braquial, bíceps braquial e abdutor curto do polegar, o que pode justificar esta divergência a respeito da afirmação de que o movimento concêntrico evidencia uma maior atividade EMG, visto que o grupo muscular em análise não possui parâmetros pré-estabelecidos, podendo apresentar resultados distintos em relação aos demais.

Em uma análise EMG comparativa entre o movimento concêntrico e excêntrico do músculo quadríceps femoral descrita por Baptista $^{34}$, não houve resultados significantes. Assim como os autores anteriores, ele destaca que diferentes grupos musculares podem apresentar-se de forma heterogênea, além de afirmar que o recrutamento de diferentes tipos de fibras musculares (contração rápida ou lenta), em diferentes ADM pode gerar dados EMG assimétricos. Tratando-se dos dados EMG mediante a utilização do Bastão de Enrolamento, foi demonstrada efetividade EMG na fase excêntrica, o que diverge dos resultados citados por este autor, afirmando não haver diferenças.
Em consideração a ADM, notou-se que durante as execuções dos testes, inevitáveis movimentos compensatórios podem ter diminuído a ativação da musculatura em análise, levando a uma menor captação EMG durante a fase concêntrica, e de certa forma facilitando o movimento e diminuindo a necessidade exclusiva da ativação da musculatura alvo (flexores ou extensores do punho), uma vez que a articulação do punho por ser distal em relação às demais dos MMSS (cotovelo e ombro), necessitava manter uma mecânica de movimento livre de influências durante a coleta.

Um movimento realizado com ampla ADM produz maior ativação muscular, resultando num recrutamento maior de unidades motoras conforme a argumentação de Paulo, Ugrinowitsch, Leite, Arsa, Marchetti e editores ${ }^{35}$. Foi notável que durante o movimento concêntrico, o enrolamento da corda apresentava-se em um ritmo mais acelerado, com menor ADM, o que pode ter diminuído a captação EMG em consequência do desgaste muscular relatado pelas voluntárias, fato que pode ser minimizado com o aperfeiçoamento dos movimentos e treinamento prévio ao início do desenvolvimento desta pesquisa.

Tratando-se da presença do desgaste muscular, não há um consenso dentre os autores quanto a sua participação e interferência na captação do sinal EMG. Enquanto alguns não validam sua influência, uma parcela aborda que a fadiga pode interferir no sinal, como citado por Moritani, Muramatso e Muro ${ }^{36}$. 
Os autores afirmam que o desgaste muscular pode alterar as ondas EMG, tornando necessário utilizar varias coletas em intervalos de ensaios separados, a fim de evitar que os registrados EMG sofram alterações e/ou influências, fato que não pode ser executado durante a aplicação deste método de pesquisa, onde as voluntárias executaram todos os testes com o Bastão de Enrolamento em um único dia em consequência de suas disponibilidades.

Bandeira, Berni e Bigaton ${ }^{24}$ divergem desta afirmação, abordando que a isquemia induzida na musculatura extensora do punho, quando relacionada à fadiga muscular e produção força, não provoca alterações EMG, e descrevem que a SEMG altera de acordo com o ambiente analisado. $O$ que pode ser observado dentre os estudos EMG é que diferentes grupos musculares, associados a ambientes biomecânicos diferenciados, apresentam dados heterogêneos, abrangendo assim os resultados presentes nesta pesquisa.

Diante de tais aspectos, tornam-se relevantes novos planejamentos em relação à utilização do Bastão de Enrolamento, uma vez que, conforme as abordagens anteriores, se aperfeiçoado o posicionamento dos MMSS, ADM e postura inicial para execução do exercício (tal como um ângulo de flexão do ombro menor que $90^{\circ}$ ), a sobrecarga exercida sobre a musculatura desta articulação pode diminuir, minimizando os déficits musculares, posturais e/ou alterações compensatórias, tornando ainda mais efetivo o objetivo de fortalecimento muscular já demostrando mediante o uso deste equipamento.

Em consequência à finalização deste estudo e expectativa do desenvolvimento e aperfeiçoamento do mesmo, os resultados EMG obtidos fornecem recursos abrangentes quanto ao conhecimento da utilização deste método alternativo de fortalecimento muscular. Vários aspectos biomecânicos podem ser analisados mediante os resultados proporcionados pela utilização deste ensaio EMG, os quais são justificados por Christou e Carlton ${ }^{37}$.

Em concordância às temáticas EMG definidas por Carpentier, Duchateau e Hainaut32, e além da ampla abordagem descrita até o momento, outro parâmetro importante e influente na avaliação dos dados EMG pode ser apresentado, tal como o potencial de tensão muscular.

Delavier $^{38}$ afirma que um grupo muscular considerado menos eficiente quanto à capacidade de gerar força, necessita de um maior recrutamento de fibras e unidades motoras a fim de superar uma determinada resistência. Não foi objetivo deste estudo fazer uma análise comparativa entre os músculos flexores e extensores do punho, entretanto, devido à ampla fonte de fenômenos EMG apresentados, tornam-se possíveis algumas relações.

Em uma abordagem individual, no teste de CMV (RMSb), o qual apresentou a máxima ativação muscular correspondente a 100\% da capacidade de força (flexores 264.1£85.1 e 
extensores 297.3 \pm 87.0 ), foi observado maior ativação do grupo extensor. Baseando-se nas argumentações de Delavier38, este grupo muscular necessitou de um maior recrutamento de fibras musculares para superar a resistência (por ser considerado menos eficiente quanto ao flexor).

Assim como no teste CMV, nos demais, todos apresentaram maior captação EMG do grupo extensor, visto que o mesmo ambiente biomecânico foi pressuposto ao grupo muscular flexor e este não necessitou de uma maior ativação EMG para superar a mesma carga.

Esta temática não produz uma afirmação concreta sobre considerar o grupo extensor como sendo menos eficiente do que o flexor. Sabe-se que o equilíbrio muscular entre os flexores e extensores do punho é essencial para a manutenção do bom funcionamento desta articulação, porém não deixa de ser um ponto de vista interessante, o qual proporciona um aprofundamento sobre esta crítica.

Como citado anteriormente a respeito dos inúmeros fatores que podem levar a dados EMG distintos e como há uma parcela dos autores que abordamas divergências dos resultados relacionados à EMG, torna-se ainda mais necessário e promissor o enriquecimento científico sobre a utilização do Bastão de Enrolamento, tendo vista os resultados desta pesquisa, justificando que o equipamento Bastão de Enrolamento trabalha a musculatura flexora e extensora do punho de maneira efetiva, mesmo contra uma carga considerada leve $(1 \mathrm{~kg})$.

\section{CONCLUSÃO}

Este estudo revelou que a utilização da análise da captação EMG mostrou ser uma ferramenta fundamental para definir e conhecer as respostas musculares durante a utilização do Bastão de Enrolamento, tendo-se observado a ativação significativa da musculatura alvo, comprovada nos resultados EMG, no tocante aos movimentos de flexão e extensão, com maior ativação muscular perante o enrolamento da corda em direção oposta ao membro analisado. Observou-se ainda que a ativação muscular foi mais evidente nas fases excêntricas dos exercícios, em ambas as direções de movimento.

Notou-se que durante a utilização deste equipamento, os dados EMG justificam a utilização do Bastão de Enrolamento com o seu propósito de fortalecimento muscular e diante da ampla abordagem sobre inúmeros aspectos que influenciam na eficácia dos dados EMG, é necessário o desenvolvimento de pesquisas futuras, associadas a variações do posicionamento inicial e adequações de ADM, com o intuito de efetivar o objetivo de fortalecimento muscular deste equipamento, assim como compara-lo a outros métodos convencionais de fortalecimento muscular para a articulação do punho, como barras, polias e alteres. 


\section{REFERENCIAS}

1. Dias J, Ovando A, Kulkamp W, Borges Junior N. Força de preensão palmar: métodos de avaliação e fatores que influenciam a medida. Rev Bras Cineamtropom Desemp Hum. 2010; 12(3) 209-2016.

2. Fernandes L, Bertoncello D, Pinheiro N, Drumond L. Correlações entre força de preensão manual e variáveis antropométricas da mão de jovens adultos. Fisiot Pesq. 2011; 18(2) 151-156.

3. Fernandes L, Araújo M, Matheus I, Medalha C, Shimano A e editores. Comparação de dois protocolos de fortalecimento para preensão palmar. Rev Bras Fisiot. 2003; 7(1) 17-23.

4. Foltran F, Silva L, Sato T, Coury H. What electrogoniometry sensor is most suitable for measuring wrist movement? Fisiot Mov. 2011; 24(2) 357-366.

5. Foltran F, Silva L, Sato T, Coury H. Wrist electrogoniometry: are current mathematical correction procedures effective in reducing crosstalk in functional assessment? Braz J Phys Ther. 2013; 17(1) 32-40.

6. Padula R, Souza V, Coury H. Tipos de preensão e movimentos do punho durante atividade de manuseio de carga. Rev Bras Fisiot. 2006; 10(1) 29-34.

7. Moreira D, Álvarez R, Gogoy J, Cambraia A. Aproach about palmar prehension usyng dynamometer JAMAR: a literature revision. Rev Bras Ci e Mov. 2003; 11(2) 95-99. 8. Sande L, Coury H. Aspectos biomecânicos e ergonômicos associados ao movimento de preensão palmar. Fisiot Pesq. 1998; 5(2) 71-82.

9. Petten A, Ávila A. Efeito do uso de órtese de punho na ativação da musculatura flexora e extensora do punho. Rev Bras Ortop. 2010; 45(1) 72-78.

10. Reis Filho A, Vieira Junior R, Voltarelli F. Avaliação do treinamento resistido de flexão e extensão do punho na força de preensão manual em mulheres de meia idade e idosas. Rev Bras Fisiot. 2014; 22(1) 87-95.

11. Sugawara L, Yanaguizawa M, Ikawa M, Takahashi $\mathrm{R}$, Natour J e editores. Carpal instability. Rev Bras Reumatol. 2008; 48(1) 34-38.

12. Silva R. Sports injuries of the upper limb. Rev bras Ortop. 2010; 45(2) 122-131.

13. Roque V, Monteiro J, Pereira F, Ramos P. Reabilitação das artroplastias totais do punho. Rev Port Ortop Traum. 2012; 20(3) 303-310.

14. Araújo V, Carvalhais V, Ocarino J, Souza T, Fonseca S. Effect of strength and stretching training on tissue passive stiffness. Fisiot Mov. 2012; 25(4) 869-882.

15. Ribeiro L, Neri A. Physical exercise, muscle strength and the day to day activities of elderly women. Ciên \& Sa Colet. 2012; 17(8) 2169-2180.

16. Coelho D, Duarte M. Parametric relationship between the center of mass identification and pressure center during quiet standing posture. Braz J Biomech. 2010; 11(20) 86-92.

17. Wattanaprakornkul D, Halaki M, Boettcher C, Cathers I, Ginn K. A comprehensive analysis of muscle recruitment patterns during shoulder flexion: an electromiographic study. Clinic Anatom. 2011; 24(1) 619-626.

18. Montgomery D. Design and analysis of Experiments. 4 ed. NY: John Wiley \& Sons; 1997.

19. Sociedade Brasileira de Cardiologia. Parâmetros hemodinâmicos de normalidade. Disponível em: http:/ / www.cardiol.br/ acesso em 01/11/2014.

20. Oliveira T, Laterza M, Ferreira R, Werneck F, Paixão $\mathrm{J}$ e editores. Effectiveness of a program of evaluation and physical exercise recommendation for women. Rev Bras Cien e Sa. 2011; 30(9) 1-8.

21. Souza J, Pasinato F, Basso D, Corrêa E, Silva A. Biophotogrammetry: reliability of measurements obtained with a posture assessment software (SAPO). Rev Bras Cineamtropom Desemp Hum. 2011; 13(4) 299-305.

22. Hermes H, Freriks B, Klug C, Rau G, Development of recommendations for SEMG sensors and sensor placement procedures. J Electrom Kinesiol. 2000; 10(1) 361-374.

23. Eletromiografia e Biofeedback Miotec. Manual do usuário: Eletromiógrafo Miotool 400. Disponível em: http://www.miotec.com.br/ acesso em 01/10/2014.

24. Bandeira C, Berni K, Bigaton D. Electromyographic analysis and strength of the wrist extensor muscle group during induced ischemia. Rev Bras Fisiot. 2009; 13(1) 31-37.

25. SENIAM- Surface Electromyography for the NonInvasive Assessment of Muscles. Recommendations of sensor locations, size and type. Disponível em: http:// www.seniam.org/ acesso em 01/10/2014.

26. Winter D. The biomechanics and motor control of human gait: nonnal, elderly and pathological. In: Winter D, organizer. University of Waterloo Press; 1991. 2 ed, p.143.

27. Knutson L, Soderberg G, Ballantyne B, Clarke W. A study ofvarious normalization procedures for within day electromyographic data. J Electrom Kinesiol. 1994; 4(1) 47-59.

28. Ervilha U, Duarte M, Amadio A. Estudo sobre procedimentos de normalização do sinal eletromiográfico durante o movimento humano. Rev Bras Fisiot. 1998; 3(1) $15-20$

29. Marchetti P, Duarte M. Instrumentação em eletromiografia [manual de utilização]. São Paulo (SP): Universidade de São Paulo, Laboratório de Biofísica; 2006.

30. Ayres M, Ayres Júnior M, Ayres D, Santos A. BioEstat: aplicacões estatísticas nas áreas das ciências biológicas e médicas. In: Ayres M, Santos A, organizadores. Duas amostras independentes. Bélem PA: Sociedade Civil Mamirauá; 2007. 1 ed, p.128.

31. Ide B, Ramari C, Muramatsu L, et al. Surface electromyography aplications in exercise physiology. Rev Acta Bras Mov Hum. 2012; 2(4) 60-78.

32. Carpentier A, Duchateau J, Hainaut K. Loaddependent muscle strategy during plantar flexion in humans. J Electrom Kinesiol. 1998; 9(1) 1-11.

33. Blackburn J, Mynark R, Padua D, Guskiewicz K. Influences of experimental factors on spinal stretch reflex latency and amplitude in the human triceps surare. J Electrom Kinesiol. 2006; 16(2) 42-50.

34. Baptista R. Adaptações neuromecânicas ao treinamento de força utilizando ações excêntricas e concêntricas em idosos saudáveis do sexo masculino [dissertação]. Porto Alegre RS: Universidade Federal do Rio Grande do Sul, Escola de Educação Física; 2011.

35. Paulo A, Ugrinowitsch C, Leite G, Arsa G, Marchetti $\mathrm{P}$ e editores. Efeito agudo dos exercícios de flexibilidade no desempenho de força máxima e resistência de força de membros inferiores e superiores. Motriz. 2012; 18(2) 345-355.

36. Moritani T, Muramatso S, Muro M. Activity of motor units during concentric and eccentric contractions. Am J Physiol Med. 1987; 66 (2) 338-350.

37. Christou E, Carlton L. Motor output is more variable during eccentric compared with concentric contractions. Med Sci Sport Exer. 2002; 34(2) 1173-1778.

38. Delavier F. Guia dos movimentos de musculação. 5 ed. São Paulo: Manole; 2013.

OBSERVAÇÃO: Os autores declaram não existir conflitos de interesse de qualquer natureza. 